\title{
Polymorphisms of Potential Drug Resistant Molecular Markers in Plasmodium Vivax From China-Myanmar Border During 2008 to 2017
}

\section{Zhen-sheng Wang}

CAMS PUMC: Chinese Academy of Medical Sciences and Peking Union Medical College

\section{Chun-yan Wei}

CAMS PUMC: Chinese Academy of Medical Sciences and Peking Union Medical College

\section{Yun-chun Pan}

CAMS PUMC: Chinese Academy of Medical Sciences and Peking Union Medical College

\section{Zhi-hua Wang}

Guilin Medical University

Xin Ji

China Academy of Chinese Medical Sciences

\section{Qianqian Chen}

CAMS PUMC: Chinese Academy of Medical Sciences and Peking Union Medical College

Lian-hui Zhang

CAMS PUMC: Chinese Academy of Medical Sciences and Peking Union Medical College

Zenglei Wang ( $\sim$ zengleiwang@pumc.edu.cn)

Chinese Academy of Medical Sciences \& Peking Union Medical College Institute of Pathogen Biology Heng Wang

CAMS PUMC: Chinese Academy of Medical Sciences and Peking Union Medical College

\section{Research Article}

Keywords: Plasmodium vivax, antimalarial drugs, drug resistance, molecular markers, China-Myanmar border

Posted Date: January 24th, 2022

DOI: https://doi.org/10.21203/rs.3.rs-1278266/v1

License: (1) (1) This work is licensed under a Creative Commons Attribution 4.0 International License. Read Full License 
Version of Record: A version of this preprint was published at Infectious Diseases of Poverty on April 25th, 2022. See the published version at https://doi.org/10.1186/s40249-022-00964-2. 


\section{Abstract}

Background: Plasmodium vivax remains the predominant species at the China-Myanmar border, imposing a major challenge to the recent gains in regional malaria elimination. To closely supervise the emerging of drug resistance in this area, we surveyed the variations in genes potentially correlated with drug resistance in $P$. vivax parasite and the possible drug selection with time.

Methods: A total of 235 P. vivax samples were collected from patients suffering uncomplicated malaria at Yingjiang, Tengchong, and Longling counties, and Nabang port in China, Yunnan province, and Laiza township in Myanmar, during 2008 to 2017. Five potential drug resistance genes were amplified utilizing nested-PCR and analyzed, including pvdhfr, pvdhps, pvmdr1, pvcrt-o, and pvk12.

Results: The $p v d h f r F 57 \mathrm{I}$ L, S58R, T61M and S117T/N presented in $40.64 \%, 56.68 \%, 40.11 \%$, and $56.04 \%$ of the sequenced $P$. vivax isolates, and these mutations significantly decreased with years. The haplotype formed by these quadruple mutations predominated at Yingjiang, Tengchong, Longling and Nabang. While a mutation H99S/R (56.59\%) dominated at Laiza and increased with time. In pvdhps, the A383G prevailed in $69.16 \%$ of the samples, which remained the most prevalent haplotype. However, a significant decrease of its occurrence was also noticed over the time. The S382A/C and A553G existed in $8.37 \%$ and $30.84 \%$ of the isolates, respectively. In pvmdr1, the mutation Y976F occurred at a low frequency in $5 / 232$ (2.16\%), while T958M was fixed and F1076L was approaching fixed (72.41\%). The K10 insertion was detected at an occurrence of $33.17 \%$ in pvcrt-o, whereas there was no significant difference among the sites or over the time. No mutation was identified in $p v k 12$.

Conclusions: Mutations related with resistance to antifolate drugs are prevalent in this area as expected, while their frequencies decrease significantly with time, suggestive of increased susceptibility of $P$. vivax parasite to antifolate drugs. Resistance to $\mathrm{CQ}$ is possibly emerging. However, since the molecular mechanisms underneath CQ resistance is yet to be better understood, close supervision of clinical drug efficiency and continuous function investigation is urgently needed to alarm drug resistance.

\section{Background}

Malaria has been a ghastly torment to human beings for millenniums and still infects 229 million people nowadays. Of the five species lead to human malaria, Plasmodium falciparum and vivax are two major causes. Africa suffers the most morbidity and mortality resulted from $P$. falciparum, while Southeast Asia accounts for the most vivax burden globally. In 2019, 51\% of the world's 6.4 million P. vivax cases occurred in Southeast Asia (World Malaria Report. Geneva: WHO; 2020). The Greater Mekong Subregion (GMS) in Southeast Asia has targeted eliminating the menace of malaria by 2030 . Of the six countries within GMS, malaria transmission levels are drastically various by country. Myanmar suffered the heaviest malaria load in 2016, whereas China reported no autochthonous malaria in 2017 and was certified indigenous malaria-free by the World Health Organization in 2021. However, China still faces the challenge of maintaining the control efforts because it shares a long border with Myanmar, involving 18 
counties in Yunnan Province. Thus, the cross-border transmission of malaria remains a great risk to China. $P$. vivax is the predominant malaria species in the China-Myanmar border area, which caused the recent malaria outbreaks in 2013 and 2016 [1]. Despite the cause of these outbreaks is ambiguous, increased drug resistance might be one of the factors. It is therefore an urgent priority to supervise drug resistance in $P$. vivax on the China-Myanmar border.

Increasing reports have focused on drug resistance of $P$. vivax mainly to chloroquine (CQ) and sulphadoxine-pyrimethamine (SP) regimens. CQ was first synthesized in 1934 and quickly proved to be a successful and important antimalarial agent [2]. It is now the most commonly administered drug for treating $P$. vivax infections. Other than the rapid resistance occurring in $P$. falciparum in 1950s, CQ resistant $P$. vivax was initially reported in late 1980s from Papua New Guinea [3] and Indonesia [4]. This was soon discovered in all around the world. High-level CQ-resistant $P$. vivax was reported in 1993 from north Myanmar [5], an area close to the southern border of China. In China, CQ has been used as the firstline drug to treat $P$. vivax along with primaquine to prevent relapse for nearly 60 years. Clinical failure or reduced efficacy of CQ-primaquine were reported in both border region [6] and the mainland [7]. SP is mainly applied in intermittent preventive treatment of malaria in pregnancy and infants, as well as in seasonal chemoprevention after its spread of resistance to $P$. falciprum. It still remains as a partner drug in artemisinin-based combination therapy (ACT) to treat sensitive $P$. falciparum. Albeit SP is not recommended to treat $P$. vivax, the common mixed-infection of $P$. falciparum and vivax in endemic area has introduced drug pressure and consequently selected SP-resistant isolates, which have been determined globally. Artemisinin resistance in $P$. vivax has not yet been reported, however, the emergence and spread of such resistance in $P$. falciparum has forewarned its appearance likely in $P$. vivax.

Molecular markers are efficient tools to monitor drug resistance. In $P$. falciparum, such markers are primarily manifested by mutant single nucleotide polymorphisms (SNPs) in related genes. CQ and SP resistance is correlated with chloroquine resistance transporter (CRT) gene and two genes encoding dihydropteroate synthase (DHPS) and dihydrofolate reductase (DHFR) enzymes, respectively, while artemisinin resistance is associated with the kelch domain of K13 gene. In addition, multidrug resistance 1 (MDR1) gene is linked to resistance to multiple antimalarials as indicated by its name. However, identification of resistant markers in $P$. vivax is particularly challenging due to the limitation of in vitro culture. Consequently, investigation of vivax resistance markers is focused on the orthologs of those identified in $P$. falciparum, mainly including $p v d h f r$, pvdhps [8-10], pvmdr1, pvcrt-o [11, 12], as well as pvk12 [13-15], the ortholog of $p f k 13$ gene. Indeed, studies have confirmed mutations F57I/L, S58R, $\mathrm{T} 61 \mathrm{M}$ and $\mathrm{S} 117 \mathrm{~N}$ in pvdhfr are responsible for pyrimethamine resistance $[9,16-18]$, while S382A/C, A383G, and A553G in pvdhps are correlated with sulfadoxine resistance [19]. Albeit polymorphisms in codons $86,184,1034,1042$ and 1246 of the pfmdr1 gene were reported to be associated with CQ resistance, only Y976F showed the association with decreased susceptibility to $\mathrm{CQ}$, as well as to mefloquine and artemisinins [20-22]. However, the correlation of pvcrt-o with CQ resistance remains ambiguous. Survey of pvcrt-o gene failed in identifying its linkage with clinical CQ treatment failure[23], whereas analysis of mutant isoforms in yeast implies mutations in pvcrt-o might change the susceptibility of vivax parasite to CQ [24]. Similarly, the copy number of pvcrt-o was found correlated with 
CQ resistance in Brazilian Amazon [25], while it was not observed in samples from Papua Indonesia [26]. Yet, the mechanism of CQ resistance still needs to be unveiled. Since the Kelch 13 (K13) gene in $P$. falciparum is considered as the main molecular marker of artemisinin resistance, likewise, its ortholog pvk12 might be selected by drug pressure.

We report here the polymorphisms of these candidate drug resistant molecular markers, $p v d h f r, p v d h p s$, pvmdr1, pvcrt-o and pvk12, in P. vivax from five sites along China-Myanmar Border during 2008 to 2017 to further understand their geographical distribution and potential selection over time.

\section{Methods}

\section{Sample collection}

Filter paper blood samples were collected from subjects attending local clinics with fever or other symptoms suggestive of malaria, during January 2008 to December 2017 at five sites on China-Myanmar border, including Yingjiang county, Tengchong county, Longling county, Nabang port in China, and Laiza township in Myanmar. The diagnosis of $P$. vivax infection was carried out by microscopic examination of Giemsa-stained thin and thick blood smears. Confirmed vivax malaria cases who consented to participate provided blood spots via standard finger-prick method. The spots were dried, numbered and sealed in plastic bags and stored at $-20^{\circ} \mathrm{C}$ for later use.

\section{Genomic DNA extraction, amplification and sequencing}

Parasite genomic DNA was extracted using the Genomic DNA Extraction Kit (NanoMagBio, China) via a 96-well high-throughput nucleic acid extraction instrument (NanoMagBio, China) in accordance with the manufacturer's instructions. Genomic DNA was eluted in $50 \mu \mathrm{l}$ of Tris- ethylene diamine tetraacetic acid (EDTA) buffer and stored at $-20^{\circ} \mathrm{C}$. Fragments of pvdhfr (755 bp), pvdhps (731 bp), pvmdr1 (64 bp), pvk12 (2108 bp) were amplified by nested PCR in $25 \mu$ reaction volumes as previously described [27-29], while pvcrt-o (1272 bp) was amplified using regular PCR. One microliter of genomic DNA was applied as template for both nested and regular PCR in each reaction. The primers used in DNA amplification were listed in Additional file 1: Table S1. The PCR products were sequenced (Beijing TsingKe Biotech Co. Ltd., Beijing, China) commercially.

\section{Sequence analysis}

The nucleotide and deduced amino acid sequences were assembled, aligned and compared to the reference sequences from the NCBI database using DNAMAN software (version 9.0.1). Mutation analyses were conducted through MEGA software (version 10.0.5, https://www.megasoftware.net/). The accession numbers of reference sequences used in this study were as follows: $p v d h f r$ (X98123), pvdhps 
(XM001617159), pvmdr1 (AY618622), pvcrt-o (AF314649), and pvk12 (PVX_083080). All sequences were submitted to GenBank (MZ960937 - MZ961394).

\section{Statistical analysis}

The percentile data were summarized utilizing Microsoft Excel 2015. The Chisq.test or Fisher's.test function in $\mathrm{R}$ language (version 3.5.3) were used to assess the frequency differences of mutations between years. Pearson's chi-square test was used to determine statistical significance $(P<0.05)$. If the data did not meet the assumptions of the chi-square test ( $80 \%$ of the cells having expected values of five or more, and no one cell having an expected value of less than one), an adjusted chi-square test and Fisher's exact test were therefore performed.

\section{Results}

\section{The origins and clinical treatment of the cases}

A total of 235 P. vivax infected patients suffering uncomplicated malaria during January 2008 to December 2017 were enrolled in this study, with 13 (5.53\%) participating during 2008-2010, 31 (13.19\%) through 2012-2015, and a majority (191, 81.28\%) between 2016 and 2017. Eighty of these samples were collected from Laiza township in Myanmar, while the rest were from four sites in Yunnan province in China, including 91 from Yingjang county, 41 from Longling county, 10 from Tengchong county and 13 from Nabang port. All patients were provided with anti-malarial therapy in accordance with the WHO guidelines for malaria treatment. Most cases $(137,58.30 \%)$ received 8-day chloroquine combined with 8day primaquine regimen, while the rest were treated with 3-day chloroquine combined with 14-day primaquine $(60,25.53 \%$ ) or a single dosage of naphthoquine phosphate (naphthoquine complexed with artemisinin, 35, 14.89\%) (Table 1). DNA from their blood samples was used to amplify the pvdhfr, pvdhps, pvmdr1, pvcrt-o and pvk12 gene sequences, and resulted in 187, 227, 232, 199, and 138 sequences. 
Table 1

General information of the enrolled cases.

\begin{tabular}{|ll|}
\hline General information & Case number (\%) \\
\hline Year & \\
\hline $2008-2010$ & $13(5.53)$ \\
\hline $2012-2015$ & $31(13.19)$ \\
\hline $2016-2017$ & $191(81.28)$ \\
\hline Gender & \\
\hline Male & $78(33.19)$ \\
\hline Female & $157(66.81)$ \\
\hline Residence & \\
\hline Laiza township & $80(34.21)$ \\
\hline Yingjiang county & $91(38.72)$ \\
\hline Longling county & $41(17.59)$ \\
\hline Tengchong county & $10(4.26)$ \\
\hline Nabang port & $13(5.53)$ \\
\hline Drug administration & \\
\hline CQ(8 days)+Primaquine(8 days) & $137(58.30)$ \\
\hline CQ(3 days)+Primaquine(14 days) & $60(25.53)$ \\
\hline Compound naphthoquine tablet (single dosage) & $35(14.89)$ \\
\hline ACTs & $1(0.43)$ \\
\hline NR & $2(0.85)$ \\
\hline NR: No records & \\
\hline
\end{tabular}

\section{Pvdhfr gene}

Seven point mutations presented in pvdhfrgene, including C49R (1.07\%), F57I/L (40.64\%), S58R (56.68\%), T61M (40.11\%), H99S/R (56.59\%), S117T/N (56.04\%), and I173F (0.55\%). Among these, the frequency of F57I/L, S58R, T61M and S117T/N decreased significantly with years, while H99S/R increased (Table 2). Haplotype analysis of pvdhfr revealed 11 distinct allelic forms, including the wild type (WT), haplotypes carrying a single mutation (58R, 99S, and 173F), double mutations (57L/58R and $58 \mathrm{R} / 117 \mathrm{~N})$, triple mutations (57L/58R/61M), quadruple mutations (57I/58R/61M/117T and 
57L/58R/61M/117T), and quintuple mutations (49R/57l/58R/61M/117T and 49R/57L/58R/61M/117T) (Table 3). Of note, the prevalence of haplotype containing a single mutation H99S differed significantly in samples across three time periods ( $0 \%$ vs $13.33 \%$ vs $33.33 \%, P=0.006)$, indicating a recent selection of this mutation in this area. 
Table 2

Prevalence of point mutations during different time periods.

\begin{tabular}{|c|c|c|c|c|}
\hline \multirow[t]{2}{*}{ Mutations" } & \multicolumn{4}{|c|}{ Number of mutations (\%) } \\
\hline & $2008-2010$ & $2012-2015$ & $2016-2017$ & Total \\
\hline pvdhfr & $n=13$ & $n=30$ & $n=144$ & $n=187$ \\
\hline C49R & $1(7.69)$ & $0(0)$ & $1(0.69)$ & $2(1.07)$ \\
\hline F57I/L & $11(84.62)$ & $18(60.00)$ & $47(32.64)^{\star \star \star}$ & $76(40.64)$ \\
\hline S58R & $12(92.31)$ & $21(70.00)$ & 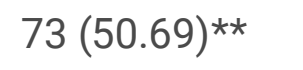 & $106(56.68)$ \\
\hline $\mathrm{T} 61 \mathrm{M}$ & $11(84.62)$ & $17(56.67)$ & $47(32.64)^{\star \star \star}$ & $75(40.11)$ \\
\hline H99S/R & $2(15.38)$ & $10(33.33)$ & $91(63.19)^{\star \star \star}$ & $103(56.59)$ \\
\hline S117T/N & $12(92.31)$ & $19(63.33)$ & $71(49.31)^{\star \star}$ & $102(56.04)$ \\
\hline I173F & $0(0)$ & $1(3.33)$ & $0(0)$ & $1(0.55)$ \\
\hline pvdhps & $n=13$ & $n=30$ & $n=184$ & $n=227$ \\
\hline A372S & $0(0)$ & $0(0)$ & $1(0.54)$ & $1(0.44)$ \\
\hline S382A/C & $2(15.38)$ & $3(10.00)$ & $14(7.61)$ & $19(8.37)$ \\
\hline A383G & $12(92.31)$ & $28(93.33)$ & $117(63.59)^{\star \star \star}$ & $157(69.16)$ \\
\hline K512E & $0(0)$ & $1(3.33)$ & $11(5.98)$ & $12(5.29)$ \\
\hline A553G & $8(61.54)$ & $16(53.33)$ & $46(25.00)^{\star \star \star}$ & $70(30.84)$ \\
\hline E571Q & $0(0)$ & $0(0)$ & $9(4.89)$ & $9(3.96)$ \\
\hline G626A & $0(0)$ & $0(0)$ & $4(2.17)$ & 4 (1.76) \\
\hline A633S & $0(0)$ & $0(0)$ & $3(1.63)$ & 3 (1.32) \\
\hline D639E & $0(0)$ & $0(0)$ & 9 (4.89) & $9(3.96)$ \\
\hline S640G & $0(0)$ & $0(0)$ & 9 (4.89) & 9 (3.96) \\
\hline A647S & $0(0)$ & 1 (3.33) & 32 (17.39) & $33(15.54)$ \\
\hline pvmdr1 & $n=13$ & $n=30$ & $n=189$ & $n=232$ \\
\hline T958M & $13(100)$ & 30 (100) & 189 (100) & $232(100)$ \\
\hline Y976F & $4(30.77)$ & 1 (3.33) & $0(0)$ & $5(2.16)$ \\
\hline
\end{tabular}

The difference in the mutations among three periods of time was calculated by Pearson's Chi-squared

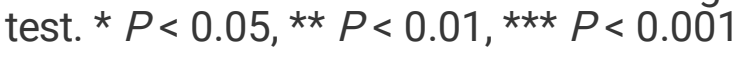

\# Point mutations are shown in boldface. 


\begin{tabular}{|c|c|c|c|c|}
\hline \multirow{2}{*}{$\begin{array}{l}\text { Mutations" } \\
\text { K997R }\end{array}$} & \multicolumn{4}{|c|}{ Number of mutations (\%) } \\
\hline & $0(0)$ & 1 (3.33) & 7 (3.70) & $8(3.45)$ \\
\hline F1076L & $8(61.54)$ & $17(56.67)$ & $143(75.66)$ & $168(72.41)$ \\
\hline pvcrt-o & $n=12$ & $n=29$ & $n=158$ & $n=199$ \\
\hline T2I & $0(0)$ & $0(0)$ & $1(0.63)$ & $1(0.50)$ \\
\hline K10 insertion & $7(58.33)$ & $11(37.93)$ & 48 (30.38) & $66(33.17)$ \\
\hline R19C & $1(8.33)$ & $0(0)$ & $0(0)$ & $1(0.50)$ \\
\hline $\mathrm{N} 57 \mathrm{H}$ & $0(0)$ & $1(3.45)$ & $0(0)$ & $1(0.50)$ \\
\hline \multicolumn{5}{|c|}{$\begin{array}{l}\text { The difference in the mutations among three periods of time was calculated by Pearson's Chi-square } \\
\text { test. } * P<0.05 \text {, } * \star P<0.01, \star \star \star P<0.001\end{array}$} \\
\hline
\end{tabular}


Table 3

Prevalence of $p v d h f r, p v d h p s, p v m d r 1$, and pvcrt-o haplotypes

\begin{tabular}{|c|c|c|c|c|c|c|}
\hline \multirow[t]{2}{*}{ Gene } & \multirow[t]{2}{*}{ Type } & \multirow[t]{2}{*}{ Haplotype } & \multicolumn{4}{|c|}{ Number of haplotype (\%) } \\
\hline & & & $\begin{array}{l}2008- \\
2010\end{array}$ & $\begin{array}{l}2012- \\
2015\end{array}$ & $\begin{array}{l}2016- \\
2017\end{array}$ & Total \\
\hline & Wild type & & $1(7.69)$ & $\begin{array}{l}4 \\
(13.33)\end{array}$ & $\begin{array}{l}23 \\
(15.97)\end{array}$ & $\begin{array}{l}28 \\
(14.97)\end{array}$ \\
\hline & $\begin{array}{l}\text { Single mutant } \\
\text { (a) }\end{array}$ & $58 \mathrm{R}$ & $0(0)$ & $0(0)$ & $2(0.69)$ & $\begin{array}{l}2 \\
(0.53)\end{array}$ \\
\hline & $\begin{array}{l}\text { Single mutant } \\
\text { (b) }\end{array}$ & 99S & $0(0)$ & $\begin{array}{l}4 \\
(13.33)\end{array}$ & $\begin{array}{l}48 \\
(33.33)^{\star \star}\end{array}$ & $\begin{array}{l}52 \\
(27.81)\end{array}$ \\
\hline & $\begin{array}{l}\text { Single mutant } \\
\text { (c) }\end{array}$ & $173 F$ & $0(0)$ & $\begin{array}{l}1 \\
(3.33)\end{array}$ & $0(0)$ & $\begin{array}{l}1 \\
(0.53)\end{array}$ \\
\hline & $\begin{array}{l}\text { Double } \\
\text { mutant }\end{array}$ & $57 \mathrm{~L} / 58 \mathrm{R}$ & $0(0)$ & $\begin{array}{l}1 \\
(3.33)\end{array}$ & $0(0)$ & $\begin{array}{l}1 \\
(0.53)\end{array}$ \\
\hline \multirow[t]{10}{*}{ pvdhfr } & $\begin{array}{l}\text { Double } \\
\text { mutant }\end{array}$ & $58 \mathrm{R} / 117 \mathrm{~N}$ & $1(7.69)$ & $\begin{array}{l}3 \\
(10.00)\end{array}$ & $\begin{array}{l}23 \\
(15.97)\end{array}$ & $\begin{array}{l}27 \\
(14.44)\end{array}$ \\
\hline & Triple mutant & $57 \mathrm{~L} / 58 \mathrm{R} / 61 \mathrm{M}$ & $0(0)$ & $\begin{array}{l}1 \\
(3.33)\end{array}$ & $0(0)$ & $\begin{array}{l}1 \\
(0.53)\end{array}$ \\
\hline & $\begin{array}{l}\text { Quadruple } \\
\text { mutant (a) }\end{array}$ & $57 \mathrm{I} / 58 \mathrm{R} / 61 \mathrm{M} / 117 \mathrm{~T}$ & $\begin{array}{l}5 \\
(38.46)\end{array}$ & $\begin{array}{l}9 \\
(30.00)\end{array}$ & $\begin{array}{l}28 \\
(19.44)\end{array}$ & $\begin{array}{l}42 \\
(22.46)\end{array}$ \\
\hline & $\begin{array}{l}\text { Quadruple } \\
\text { mutant (b) }\end{array}$ & 57L/58R/61M/117T & $5(38.46)$ & $\begin{array}{l}7 \\
(23.33)\end{array}$ & $\begin{array}{l}18 \\
(12.50) *\end{array}$ & $\begin{array}{l}30 \\
(16.04)\end{array}$ \\
\hline & $\begin{array}{l}\text { Quintuple } \\
\text { mutant (a) }\end{array}$ & 49R/57I/58R/61M/117T & $0(0)$ & $0(0)$ & $1(0.69)$ & $\begin{array}{l}1 \\
(0.53)\end{array}$ \\
\hline & $\begin{array}{l}\text { Quintuple } \\
\text { mutant (b) }\end{array}$ & 49R/57L/58R/61M/117T & $1(7.69)$ & $0(0)$ & $0(0)$ & $\begin{array}{l}1 \\
(0.53)\end{array}$ \\
\hline & Wild type & & $1(7.69)$ & $\begin{array}{l}1 \\
(3.33)\end{array}$ & $\begin{array}{l}30 \\
(16.30)\end{array}$ & $\begin{array}{l}32 \\
(14.10)\end{array}$ \\
\hline & $\begin{array}{l}\text { Single mutant } \\
\text { (a) }\end{array}$ & $383 G$ & $\begin{array}{l}4 \\
(30.77)\end{array}$ & $\begin{array}{l}7 \\
(23.33)\end{array}$ & $\begin{array}{l}63 \\
(34.24)\end{array}$ & $\begin{array}{l}74 \\
(32.60)\end{array}$ \\
\hline & $\begin{array}{l}\text { Single mutant } \\
\text { (b) }\end{array}$ & $553 G$ & $0(0)$ & $0(0)$ & $1(0.54)$ & $\begin{array}{l}1 \\
(0.44)\end{array}$ \\
\hline & $\begin{array}{l}\text { Single mutant } \\
\text { (c) }\end{array}$ & $626 \mathrm{~A}$ & $0(0)$ & $\begin{array}{l}3 \\
(10.00)\end{array}$ & $1(0.54)$ & $\begin{array}{l}4 \\
(1.76)\end{array}$ \\
\hline
\end{tabular}

The difference in the major haplotypes among three periods of time was calculated by corrected Pearson's Chi-squared test or Fisher exact test. ${ }^{*} P<0.05$, ${ }^{\star \star} P<0.01, \star \star \star P<0.001$.

a _ indicates no K10 insertion. 


\begin{tabular}{|c|c|c|c|c|c|c|}
\hline \multirow[t]{2}{*}{ Gene } & \multirow{2}{*}{$\begin{array}{l}\text { Type } \\
\text { Single mutant } \\
\text { (d) }\end{array}$} & \multirow{2}{*}{$\begin{array}{l}\text { Haplotype } \\
647 S\end{array}$} & \multicolumn{4}{|c|}{ Number of haplotype (\%) } \\
\hline & & & $0(0)$ & $\begin{array}{l}1 \\
(3.33)\end{array}$ & $\begin{array}{l}31 \\
(16.84)\end{array}$ & $\begin{array}{l}32 \\
(14.10)\end{array}$ \\
\hline & $\begin{array}{l}\text { Double } \\
\text { mutant (a) }\end{array}$ & $382 A / 383 G$ & $0(0)$ & $\begin{array}{l}2 \\
(6.66)\end{array}$ & $0(0) *$ & $\begin{array}{l}2 \\
(0.88)\end{array}$ \\
\hline \multirow[t]{12}{*}{ pvdhps } & $\begin{array}{l}\text { Double } \\
\text { mutant (b) }\end{array}$ & $383 G / 553 G$ & $\begin{array}{l}6 \\
(46.15)\end{array}$ & $\begin{array}{l}14 \\
(46.67)\end{array}$ & $\begin{array}{l}30 \\
(16.30) \star \star \star\end{array}$ & $\begin{array}{l}50 \\
(22.03)\end{array}$ \\
\hline & $\begin{array}{l}\text { Double } \\
\text { mutant (c) }\end{array}$ & $512 \mathrm{E} / 647 \mathrm{~S}$ & $0(0)$ & $0(0)$ & $1(0.54)$ & $\begin{array}{l}1 \\
(0.44)\end{array}$ \\
\hline & $\begin{array}{l}\text { Double } \\
\text { mutant (d) }\end{array}$ & $626 \mathrm{~A} / 633 \mathrm{~S}$ & $0(0)$ & $0(0)$ & $2(1.09)$ & $\begin{array}{l}2 \\
(0.88)\end{array}$ \\
\hline & $\begin{array}{l}\text { Triple mutant } \\
\text { (a) }\end{array}$ & $372 S / 626 \mathrm{~A} / 647 \mathrm{~S}$ & $0(0)$ & $0(0)$ & $1(0.54)$ & $\begin{array}{l}1 \\
(0.44)\end{array}$ \\
\hline & $\begin{array}{l}\text { Triple mutant } \\
\text { (b) }\end{array}$ & $382 A / 383 G / 553 G$ & $\begin{array}{l}2 \\
(15.38)\end{array}$ & $\begin{array}{l}1 \\
(3.33)\end{array}$ & $5(2.72)$ & $\begin{array}{l}8 \\
(3.52)\end{array}$ \\
\hline & $\begin{array}{l}\text { Triple mutant } \\
\text { (c) }\end{array}$ & $383 G / 512 E / 553 G$ & $0(0)$ & $\begin{array}{l}1 \\
(3.33)\end{array}$ & $1(0.54)$ & $\begin{array}{l}2 \\
(0.88)\end{array}$ \\
\hline & $\begin{array}{l}\text { Quadruple } \\
\text { mutant (a) }\end{array}$ & $382 \mathrm{C} / 383 \mathrm{G} / 512 \mathrm{E} / 553 \mathrm{G}$ & $0(0)$ & $0(0)$ & $9(4.86)$ & $\begin{array}{l}9 \\
(3.96)\end{array}$ \\
\hline & $\begin{array}{l}\text { Quadruple } \\
\text { mutant (b) }\end{array}$ & $383 G / 571 Q / 639 E / 640 G$ & $0(0)$ & $0(0)$ & $9(4.89)$ & $\begin{array}{l}9 \\
(3.96)\end{array}$ \\
\hline & Wild type & & $0(0)$ & $0(0)$ & $0(0)$ & $0(0)$ \\
\hline & $\begin{array}{l}\text { Single mutant } \\
\text { (a) }\end{array}$ & $958 \mathrm{M}$ & $\begin{array}{l}5 \\
(38.46)\end{array}$ & $\begin{array}{l}12 \\
(40.00)\end{array}$ & $\begin{array}{l}39 \\
(20.63)\end{array}$ & $\begin{array}{l}56 \\
(24.14)\end{array}$ \\
\hline & $\begin{array}{l}\text { Double } \\
\text { mutant (a) }\end{array}$ & $958 \mathrm{M} / 1076 \mathrm{~L}$ & $\begin{array}{l}4 \\
(30.77)\end{array}$ & $\begin{array}{l}16 \\
(53.33)\end{array}$ & $\begin{array}{l}143 \\
(75.66)^{\star \star \star}\end{array}$ & $\begin{array}{l}162 \\
(69.83)\end{array}$ \\
\hline & $\begin{array}{l}\text { Double } \\
\text { mutant (b) }\end{array}$ & 958M/997R & $0(0)$ & $\begin{array}{l}1 \\
(3.33)\end{array}$ & $6(3.17)$ & $\begin{array}{l}7 \\
(3.01)\end{array}$ \\
\hline \multirow[t]{3}{*}{ pvmdr1 } & $\begin{array}{l}\text { Triple mutant } \\
\text { (a) }\end{array}$ & $958 \mathrm{M} / 976 \mathrm{~F} / 1076 \mathrm{~L}$ & $\begin{array}{l}4 \\
(30.77)\end{array}$ & $\begin{array}{l}1 \\
(3.33)\end{array}$ & $0(0)$ & $\begin{array}{l}5 \\
(2.16)\end{array}$ \\
\hline & $\begin{array}{l}\text { Triple mutant } \\
\text { (b) }\end{array}$ & $958 \mathrm{M} / 997 \mathrm{R} / 1076 \mathrm{~L}$ & $0(0)$ & $0(0)$ & $1(0.53)$ & $\begin{array}{l}1 \\
(0.43)\end{array}$ \\
\hline & Wild type & & $\begin{array}{l}5 \\
(41.67)\end{array}$ & $\begin{array}{l}18 \\
(62.07)\end{array}$ & $\begin{array}{l}110 \\
(69.62)\end{array}$ & $\begin{array}{l}133 \\
(66.83)\end{array}$ \\
\hline
\end{tabular}

The difference in the major haplotypes among three periods of time was calculated by corrected

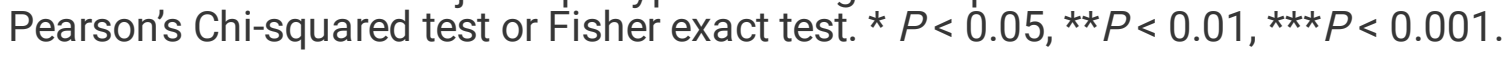

a _ indicates no K10 insertion. 


\begin{tabular}{|c|c|c|c|c|c|c|}
\hline \multirow[t]{2}{*}{ Gene } & \multirow{2}{*}{$\begin{array}{l}\text { Type } \\
\text { Single mutant } \\
\text { (a) }\end{array}$} & \multirow{2}{*}{$\begin{array}{l}\text { Haplotype } \\
\text { K10(in) }\end{array}$} & \multicolumn{4}{|c|}{ Number of haplotype (\%) } \\
\hline & & & $\begin{array}{l}6 \\
(50.00)\end{array}$ & $\begin{array}{l}10 \\
(34.48)\end{array}$ & $\begin{array}{l}47 \\
(29.75)\end{array}$ & $\begin{array}{l}63 \\
(31.66)\end{array}$ \\
\hline \multirow[t]{3}{*}{ pvcrt-o } & $\begin{array}{l}\text { Single mutant } \\
\text { (b) }\end{array}$ & K10(in) $/ 57 \mathrm{H}$ & $0(0)$ & $\begin{array}{l}1 \\
(3.45)\end{array}$ & $0(0)$ & $\begin{array}{l}1 \\
(0.50)\end{array}$ \\
\hline & $\begin{array}{l}\text { Single mutant } \\
\text { (c) }\end{array}$ & K10(in)/19C & $1(5.08)$ & $0(0)$ & $0(0)$ & $\begin{array}{l}1 \\
(0.50)\end{array}$ \\
\hline & $\begin{array}{l}\text { Single mutant } \\
\text { (d) }\end{array}$ & 2l/K10(in) & $0(0)$ & $0(0)$ & $1(0.63)$ & $\begin{array}{l}1 \\
(0.50)\end{array}$ \\
\hline \multicolumn{7}{|c|}{$\begin{array}{l}\text { The difference in the major haplotypes among three periods of time was calculated by corrected } \\
\text { Pearson's Chi-squared test or Fisher exact test. } * P<0.05,{ }^{\star} P<0.01 \text {, }{ }^{\star \star} P<0.001 \text {. }\end{array}$} \\
\hline
\end{tabular}

The geographical haplotypes prevalence analysis demonstrated that the single mutant H99S was dominant at Laiza, while it was nearly absent from other sites. However, the haplotype harboring the quadruple mutations 57//58R/61M/117T predominated in samples from Yingjiang, Tengchong and Longling. Its related haplotype with mutations 57L/58R/61M/117T prevailed in isolates from Nabang (Fig. 1a).

Three tandem repeat variations were detected in pvdhfrat positions 82 - 109. Type I was identical to the Sal I reference gene, type II contained a H99S mutation, and type III had a six amino-acid deletion at position 98-103 (THGGDN) (Fig. 1b). The prevalence of type I was significantly decreased with years, and vice versa for type II. Type III tended to increase over time, though without statistically evidence (Fig. 1c). Nevertheless, type Il prevailed in Laiza but nearly absent from other regions in China (Fig. 1d).

\section{Pvdhps gene}

Alignment of the fragments of pvdhps gene discovered 11 mutations, including A372S, S382A/C, A383G, K512E, A553G, E571Q, G626A, A633S, D639E, S640G, and A647S. However, only two variants, A383G and A553G, were prevalent in the samples at an occurrence of $69.16 \%$ and $30.84 \%$, respectively. The rest occurred at a relatively low frequency. The frequencies of A383G and A553G mutations significantly decreased with time (Table 2). This created 14 haplotypes containing WT and allelic forms harboring four single mutations (383G, 553G, 626A and 647S), four double mutations (382A/383G, 383G/553G, $512 \mathrm{E} / 647 \mathrm{~S}$ and $626 \mathrm{~A} / 633 \mathrm{~S}$ ), three triple mutations (372S/626A/647S, 382A/383G/553G and $383 \mathrm{G} / 512 \mathrm{E} / 553 \mathrm{G}$ ), and two quadruple mutations (382C/383G/512E/553G and 383G/571Q/639E/640G). As expected, the haplotype with the single mutation $\mathrm{A} 383 \mathrm{G}$ was the most prevalent one (32.60\%), followed by A383G/A553G double mutations (22.03\%) among all the $P$. vivax isolates. Interestingly, unlike the haplotype with only A383G mutation, the frequency of the haplotype with A383G/A553G significantly 
decreased with time (Table 4). By site, A383G remained the predominated haplotype in samples from Tengchong and Yingjiang, and A383G/A553G prevailed in Tengchong and Longling (Fig. 2a).

Three tandem repeat variant types at position 603-637 were identified with a variable number of repeat unit ' $G(E / D)(G / A) K L T N$ ', much less than those reported previously in the same region [30]. Type a was identical to the Sal I reference sequence and carried five repeat units, type $b$ had six units while type $c$ held only three units (Fig. 2b). No statistical difference was found in the occurrence of these tandem repeat variations with years (Fig. 2c), however, type a remained the most common one in all sites, followed by type b (Fig. 2d).

\section{Pvmdr1 gene}

Four mutations at T958M, Y976F, K997R, and F1076L were observed in pvmdr1 gene. The T958M mutation was fixed in all sequenced isolates, and the F1076L mutation was approaching fixed, occurring in $72.41 \%$ of the isolates. In contrast, Y976F and K997R happened in 5/232 (2.16\%) and 8/232 (3.45\%) (Table 2). This gave rise to five haplotypes comprised by a single mutation (958M), two double mutations (958M/1076L and 958M/997R), and two triple mutations (958M/976F/1076L and 958M/997R/1076L) (Table 5). It was noteworthy that the prevalence of the T958M/F1076L significantly increased over the three collection periods ( $30.77 \%$ vs $57.33 \%$ vs $75.66 \%, P=0.002$ ). Given that T958M was fixed in these samples, yet a recent selection of $\mathrm{F} 1076 \mathrm{~L}$ might have taken place in $P$. vivax in this region. Unsurprisingly, this $958 \mathrm{M} / 1076 \mathrm{~L}$ haplotype dominated in all of the sampling sites (Fig. 3a).

\section{Pvcrt-o gene}

Three mutations at codons 2, 19 and 57 (T2I, R19C and N57H) were detected and each occurred in one clinical isolate (Table 2). In addition, a K10 insertion was detected in $33.17 \%$ of the sequenced isolates. Thus, WT was the most prevalent haplotype (66.83\%), followed by the K10 insertion $(33.17 \%)$ among all sequenced $P$. vivax isolates (Fig. 3b).

\section{Pvk12 gene}

The 2108-bp amplified pvk12 gene fragment contained $98.55 \%$ of the coding region. Hitherto, this gene remained conserved and no polymorphism was detected from $P$. vivax in this area.

\section{Discussion}

China has achieved the goal set in the National Malaria Elimination Action Plan (2010-2020) and eliminated indigenous malaria since 2017. However, border malaria remains a great concern because the risk for malaria reintroduction from the neighboring countries still exists. In particular, malaria along the China-Myanmar border area needs to be closely supervised due to the complex geographic conditions and migrant population. Antimalarial drug resistance could also jeopardize the recent gains. This study spotlighted the drug resistance in $P$. vivax at China-Myanmar border and investigated the polymorphisms 
in those potential drug resistance genes to better understand their epidemic pattern, geographical distribution and possible recent selection.

The spread of antifolate drug resistance in $P$. vivax parasite from GMS has been widely reported and associated with point mutations in pvdhfr and pvdhps [10,31,32]. As expected, results from this study demonstrated high level resistance of viva $x$ parasite to SP at China-Myanmar border. For $p v d h f r$ gene, mutations F57I/L, S58R, T61M, S117N/T and I173L/F confer resistance to pyrimethamine [33]. Among these, S58R and S117N/T occur most frequently in highly resistant $P$. vivax, particularly, S117N/T is regarded as a drug resistance determinant [17]. The quadruple mutations F57(I/L)/S58R/T61M/S117T are likely related to high level pyrimethamine resistance and clinical treatment failure [34]. Our results presented that S58R and S117N/T mutations prevailed in $56.68 \%$ and $56.04 \%$ of the isolates, similar to those reported at the same region [30], but much lower than that detected in other areas of Myanmar (71-90\%) [28] and Thailand border (100\%) [27]. However, the frequency of these two mutations as well as the haplotype of F57L/S58R/T61M/S117T decreased significantly with time. It is possible that the susceptibility of $P$. vivax parasite to SP has increased after its withdrawal to treat the falciparum parasites which often co-infect the patients. The role of tandem repeat variation in pvdhfr remains ambiguous. Some reports indicate the sensitivity of vivax parasite to pyrimethamine is not related to the tandem repeats [35], because these variations occur outside of the substrate binding site [36]. Conversely, other studies report that a type of tandem repeat with four units might correlate with pyrimethamine drug resistance because of their co-existing with the quadruple 57I/58R/61M/117T mutations [37, 38]. Such type, however, was not found in this study.

Mutations S382A/C, A383G, and A553G in pvdhps are correlated with sulfadoxine resistance. Amongst these, mutations $\mathrm{A} 383 \mathrm{G}$ and $\mathrm{A} 553 \mathrm{G}$ might contribute more to the resistance, because they could cause a reduction in binding of sulfadoxine to $p v d h p s$ as confirmed in transgenic rodent parasites expressing Pvdhps protein $[19,39]$. Here, we found A383G and A553G were prevailed in $69.16 \%$ and $30.84 \%$ of the isolates, respectively, similar to that reported by other studies [30]. Nevertheless, the prevalence of these two mutations significantly decreased with time, which could be another sign of increasing sensitivity of $P$. vivax parasites to $\mathrm{SP}$ in this region.

Recent studies monitored a decline in the therapeutic responses of $P$. vivax malaria to CQ treatment along China-Myanmar border [40, 41]. However, the molecular mechanism underlying CQ resistance in $P$. vivax remains to be fully understood. The genes pvmdr1 and pvcrt-o might be involved in CQ resistance [42]. In pvmdr1, the in vitro sensitivity of $P$. vivax isolates carrying the Y976F shows a decrease to CQ $[20,21,43]$. This mutation is associated with CQ monotherapy failure as well [43]. The prevalence of Y976F is approaching fixation in vivax isolates from Indonesian Papua, however, here it was only detected in $2.16 \%$ of the samples, which is consistent with previous research in the same area [30]. The T958M mutation is fixed in this area, while it is confirmed irrelevant to the CQ resistance [30]. Likewise, few evidence supports the $\mathrm{F} 1076 \mathrm{~L}$ single mutation as a possible CQ resistance marker. The double mutations Y976F and F1076 L are largely found from areas with $C Q$ resistance, whereas the $F 1076 \mathrm{~L}$ single mutation is more frequent in areas with rare CQ resistance [42, 44]. It is of interest that the double mutations Y976F 
and F1076L were found only in samples from Tengchong and Longling in our study, alarming the emerging of CQ drug resistance in this border region.

The function of pvcrt-o gene in CQ resistance still needs to be fully understood. The lysine $(K)$ insertion at codon 10 might be linked with decreased CQ sensitivity [7, 20]. K10 was observed in $33.17 \%$ of the sequenced samples here, while there was no significant difference among the sites or over the time. The copy number of pvcrt-o might be another factor affecting CQ treatment, which was found to be correlated with CQ resistance in Brazilian Amazon [25], but this was not observed in Papua Indonesia [26]. The expression level of pvcrt-o was not evaluated because dried blood spots were employed in the study. Yet, close supervision of the efficacy of CQ-primaquine regimen is important to monitor CQ resistance in this region.

Mutations in the propeller domain of $p f k 13$ are associated with reduced sensitivity to artemisinin and its derivatives in $P$. falciparum $[45,46]$. Possibly, its orthologous gene in $P$. vivax parasite, $p v k 12$, would be selected with artemisinin pressure. Hitherto, the $p v k 12$ gene remains conserved in this region, and only a few non-synonymous mutations were identified recently [47]. No mutation was detected in our samples, which is consistent with previous studies $[15,27]$. Since CQ-primaquine remains the primary regimen to treat $P$. vivax in this area, artemisinin selection might have not yet developed.

\section{Conclusions}

This study reported the epidemic pattern and geographical distribution of five potential drug resistant markers, $p v d h f r, p v d h p s, p v m d r 1, p v c r t-o$ and $p v k 12$, in $P$. vivax isolates from five sites along the ChinaMyanmar border during a 10-year period. Resistance to antifolate drugs is presented as expected in this area, however, the prevalence of certain mutations related to drug resistance decreased with time. Resistance to $\mathrm{CQ}$ is possibly emerging, therefore, close supervision of clinical drug efficiency and continuous function investigation is needed to better alarm drug resistance and direct vivax malaria treatment.

\section{Abbreviations}

P. vivax. Plasmodium vivax, pvdhfr. P. vivax dihydrofolate reductase gene; pvdhps. $P$. vivax dihydropteroate synthase gene; $p v m d r 1$ : $P$. vivax multidrug resistance 1 gene; $p v c r t-0$. $P$. vivax chloroquine resistance transporter-o gene; pvk12. P. vivax kelch 12 gene; CQ: chloroquine; SP: sulphadoxinepyrimethamine; ACT: artemisinin-based combination therapy; WHO: World Health Organization; GMS: Greater Mekong Subregion; SNPs: single nucleotide polymorphisms; CRT: chloroquine resistance transporter; DHPS: dihydropteroate synthase; DHFR: dihydrofolate reductase; MDR1: multidrug resistance 1; PCR: polymerase chain reaction;

\section{Declarations}




\section{Ethics approval and consent to participate}

This study was approved by the Ethical Committee of the Institute of Pathogen Biology, Chinese Academy of Medical Sciences (IPB-2017-9).

\section{Consent for publication}

Not applicable.

\section{Availability of data and materials}

All sequences generated and analyzed during the current study were submitted to the GenBank (MZ960937 - MZ961394).

\section{Competing interests}

The authors declare that they have no competing interests.

\section{Funding}

This work has been supported by Major National Science and Technology Program of China for Innovative Drug (2017ZX09101002-001-001), CAMS Innovation Fund for Medical Sciences (CIFMS) (2017-I2M-3-016), and NHC Key Laboratory of Echinococcosis Prevention and Control (Project No.2020WZK2009).

\section{Authors' contributions}

WZS, WCY, PYC, WZH, JX, CQQ, ZLH conceived and designed the experiments. WZS, ZLH, JX, CQQ performed the experiments. WZS, WZL analyzed the data. WZS, WZL, WH wrote the manuscript. All authors read and approved the final manuscript.

\section{Acknowledgments}

Not applicable.

\section{References}

1. Geng J, Malla P, Zhang J, Xu S, Li C, Zhao Y, et al. Increasing trends of malaria in a border area of the Greater Mekong Subregion. Malar J. 2019;18(1):309.

2. Wellems TE, Plowe CV. Chloroquine-resistant malaria. J Infect Dis. 2001;184(6):770-6.

3. Rieckmann KH, Davis DR, Hutton DC. Plasmodium vivax resistance to chloroquine? Lancet. 1989;2(8673):1183-4.

4. Baird JK, Basri H, Purnomo, Bangs MJ, Subianto B, Patchen LC, et al. Resistance to chloroquine by Plasmodium vivax in Irian Jaya, Indonesia. Am J Trop Med Hyg. 1991;44(5):547-52. 
5. Myat Phone K, Myint O, Myint L, Thaw Z, Kyin Hla A, Nwe Nwe Y. Emergence of chloroquine-resistant Plasmodium vivax in Myanmar (Burma). Trans R Soc Trop Med Hyg. 1993;87(6):687.

6. Liang GL, Sun XD, Wang J, Zhang ZX. [Sensitivity of Plasmodium vivax to chloroquine in Laza City, Myanmar]. Zhongguo Ji Sheng Chong Xue Yu Ji Sheng Chong Bing Za Zhi. 2009;27(2):175-6.

7. Lu F, Lim CS, Nam DH, Kim K, Lin K, Kim TS, et al. Genetic polymorphism in pvmdr1 and pvcrt-o genes in relation to in vitro drug susceptibility of Plasmodium vivax isolates from malaria-endemic countries. Acta Trop. 2011;117(2):69-75.

8. Chulay JD, Watkins WM, Sixsmith DG. Synergistic antimalarial activity of pyrimethamine and sulfadoxine against Plasmodium falciparum in vitro. Am J Trop Med Hyg. 1984;33(3):325-30.

9. de Pecoulas PE, Tahar R, Ouatas T, Mazabraud A, Basco LK. Sequence variations in the Plasmodium vivax dihydrofolate reductase-thymidylate synthase gene and their relationship with pyrimethamine resistance. Mol Biochem Parasitol. 1998;92(2):265-73.

10. Imwong M, Pukrittayakamee S, Cheng Q, Moore C, Looareesuwan S, Snounou G, et al. Limited polymorphism in the dihydropteroate synthetase gene (dhps) of Plasmodium vivax isolates from Thailand. Antimicrob Agents Chemother. 2005;49(10):4393-5.

11. Nomura T, Carlton JM, Baird JK, del Portillo HA, Fryauff DJ, Rathore D, et al. Evidence for different mechanisms of chloroquine resistance in 2 Plasmodium species that cause human malaria. J Infect Dis. 2001;183(11):1653-61.

12. Rungsihirunrat K, Muhamad P, Chaijaroenkul W, Kuesap J, Na-Bangchang K. Plasmodium vivax drug resistance genes; Pvmdr1 and Pvcrt-o polymorphisms in relation to chloroquine sensitivity from a malaria endemic area of Thailand. Korean J Parasitol. 2015;53(1):43-9.

13. Popovici J, Kao S, Eal L, Bin S, Kim S, Menard D. Reduced polymorphism in the Kelch propeller domain in Plasmodium vivax isolates from Cambodia. Antimicrob Agents Chemother. 2015;59(1):730-3.

14. Wang M, Siddiqui FA, Fan Q, Luo E, Cao Y, Cui L. Limited genetic diversity in the PvK12 Kelch protein in Plasmodium vivax isolates from Southeast Asia. Malar J. 2016;15(1):537.

15. Deng S, Ruan Y, Bai Y, Hu Y, Deng Z, He Y, et al. Genetic diversity of the Pvk12 gene in Plasmodium vivax from the China-Myanmar border area. Malar J. 2016;15(1):528.

16. Imwong M, Pukrittakayamee S, Looareesuwan S, Pasvol G, Poirriez J, White NJ, et al. Association of genetic mutations in Plasmodium vivax dhfr with resistance to sulfadoxine-pyrimethamine: Geographical and clinical correlates. Antimicrob Agents Ch. 2001;45(11):3122-7.

17. Imwong M, Pukrittayakamee S, Renia L, Letourneur F, Charlieu JP, Leartsakulpanich U, et al. Novel point mutations in the dihydrofolate reductase gene of Plasmodium vivax. evidence for sequential selection by drug pressure. Antimicrob Agents Chemother. 2003;47(5):1514-21.

18. Tjitra E, Baker J, Suprianto S, Cheng Q, Anstey NM. Therapeutic efficacies of artesunate-sulfadoxinepyrimethamine and chloroquine-sulfadoxine-pyrimethamine in vivax malaria pilot studies: Relationship to Plasmodium vivax dhfr mutations. Antimicrob Agents Ch. 2002;46(12):3947-53. 
19. Korsinczky M, Fischer K, Chen NH, Baker J, Rieckmann K, Cheng Q. Sulfadoxine resistance in Plasmodium vivax is associated with a specific amino acid in dihydropteroate synthase at the putative sulfadoxine-binding site. Antimicrob Agents Ch. 2004;48(6):2214-22.

20. Suwanarusk R, Russell B, Chavchich M, Chalfein F, Kenangalem E, Kosaisavee V, et al. Chloroquine resistant Plasmodium vivax: in vitro characterisation and association with molecular polymorphisms. PLoS One. 2007;2(10):e1089.

21. Suwanarusk R, Chavchich M, Russell B, Jaidee A, Chalfein F, Barends M, et al. Amplification of pvmdr1 associated with multidrug-resistant Plasmodium vivax. J Infect Dis. 2008;198(10):1558-64.

22. del Carmen R, Vargas-Rodriguez M, Bastos MD, Menezes MJ, Orjuela-Sanchez P, Ferreira MU. SingleNucleotide Polymorphism and Copy Number Variation of the Multidrug Resistance-1 Locus of Plasmodium vivax. Local and Global Patterns. Am J Trop Med Hyg. 2012;87(5):813-21.

23. Nomura T, Carlton JMR, Baird JK, del Portillo HA, Fryauff DJ, Rathore D, et al. Evidence for different mechanisms of chloroquine resistance in 2 Plasmodium species that cause human malaria. Journal of Infectious Diseases. 2001;183(11):1653-61.

24. Hassett MR, Riegel BE, Callaghan PS, Roepe PD. Analysis of Plasmodium vivax Chloroquine Resistance Transporter Mutant Isoforms. Biochemistry-Us. 2017;56(41):5615-22.

25. Melo GC, Monteiro WM, Siqueira AM, Silva SR, Magalhaes BML, Alencar ACC, et al. Expression Levels of pvcrt-o and pvmdr-1 Are Associated with Chloroquine Resistance and Severe Plasmodium vivax Malaria in Patients of the Brazilian Amazon. PLoS One. 2014;9(8).

26. Pava Z, Handayuni I, Wirjanata G, To S, Trianty L, Noviyanti R, et al. Expression of Plasmodium vivax crt-o Is Related to Parasite Stage but Not Ex Vivo Chloroquine Susceptibility. Antimicrob Agents Ch. 2016;60(1):361-7.

27. Tantiamornkul K, Pumpaibool T, Piriyapongsa J, Culleton R, Lek-Uthai U. The prevalence of molecular markers of drug resistance in Plasmodium vivax from the border regions of Thailand in 2008 and 2014. Int J Parasitol Drugs Drug Resist. 2018;8(2):229-37.

28. Nyunt MH, Han JH, Wang B, Aye KM, Aye KH, Lee SK, et al. Clinical and molecular surveillance of drug resistant vivax malaria in Myanmar (2009-2016). Malar J. 2017;16(1):117.

29. Liu H, Yang HL, Tang LH, Li XL, Huang F, Wang JZ, et al. Monitoring Plasmodium vivax chloroquine sensitivity along China-Myanmar border of Yunnan Province, China during 2008-2013. Malar J. 2014;13:364.

30. Zhao Y, Wang L, Soe MT, Aung PL, Wei H, Liu Z, et al. Molecular surveillance for drug resistance markers in Plasmodium vivax isolates from symptomatic and asymptomatic infections at the ChinaMyanmar border. Malar J. 2020;19(1):281.

31. Huang B, Huang S, Su XZ, Tong X, Yan J, Li H, et al. Molecular surveillance of pvdhfr, pvdhps, and pvmdr-1 mutations in Plasmodium vivax isolates from Yunnan and Anhui provinces of China. Malar J. 2014;13:346.

32. Hawkins VN, Auliff A, Prajapati SK, Rungsihirunrat K, Hapuarachchi HC, Maestre A, et al. Multiple origins of resistance-conferring mutations in Plasmodium vivax dihydrofolate reductase. Malar J. 
2008;7:72.

33. Hawkins VN, Joshi H, Rungsihirunrat K, Na-Bangchang K, Sibley $\mathrm{CH}$. Antifolates can have a role in the treatment of Plasmodium vivax. Trends Parasitol. 2007;23(5):213-22.

34. Hastings MD, Porter KM, Maguire JD, Susanti I, Kania W, Bangs MJ, et al. Dihydrofolate reductase mutations in Plasmodium vivax from Indonesia and therapeutic response to sulfadoxine plus pyrimethamine. J Infect Dis. 2004;189(4):744-50.

35. Asih PB, Marantina SS, Nababan R, Lobo NF, Rozi IE, Sumarto W, et al. Distribution of Plasmodium vivax pvdhfr and pvdhps alleles and their association with sulfadoxine-pyrimethamine treatment outcomes in Indonesia. Malar J. 2015;14:365.

36. Kongsaeree P, Khongsuk P, Leartsakulpanich U, Chitnumsub P, Tarnchompoo B, Walkinshaw MD, et al. Crystal structure of dihydrofolate reductase from Plasmodium vivax. pyrimethamine displacement linked with mutation-induced resistance. Proc Natl Acad Sci U S A. 2005;102(37):13046-51.

37. Sharifi-Sarasiabi K, Haghighi A, Kazemi B, Taghipour N, Mojarad EN, Gachkar L. MOLECULAR SURVEILLANCE OF Plasmodium vivax AND Plasmodium falciparum DHFR MUTATIONS IN ISOLATES FROM SOUTHERN IRAN. Rev Inst Med Trop Sao Paulo. 2016;58:16.

38. Prajapati SK, Joshi H, Dev V, Dua VK. Molecular epidemiology of Plasmodium vivax anti-folate resistance in India. Malar J. 2011;10:102.

39. Pornthanakasem W, Riangrungroj P, Chitnumsub P, Ittarat W, Kongkasuriyachai D, Uthaipibull C, et al. Role of Plasmodium vivax Dihydropteroate Synthase Polymorphisms in Sulfa Drug Resistance. Antimicrob Agents Chemother. 2016;60(8):4453-63.

40. Yuan LL, Wang Y, Parker DM, Gupta B, Yang ZQ, Liu HE, et al. Therapeutic Responses of Plasmodium vivax Malaria to Chloroquine and Primaquine Treatment in Northeastern Myanmar. Antimicrob Agents Ch. 2015;59(2):1230-5.

41. Xu SL, Zeng WL, Mbenda HGN, Liu HE, Chen X, Xiang Z, et al. Efficacy of directly-observed chloroquine-primaquine treatment for uncomplicated acute Plasmodium vivax malaria in northeast Myanmar: A prospective open-label efficacy trial. Travel Med Infect Di. 2020;36.

42. Lu F, Wang B, Cao J, Sattabongkot J, Zhou H, Zhu G, et al. Prevalence of drug resistance-associated gene mutations in Plasmodium vivax in Central China. Korean J Parasitol. 2012;50(4):379-84.

43. Marfurt J, de Monbrison F, Brega S, Barbollat L, Muller I, Sie A, et al. Molecular markers of in vivo Plasmodium vivax resistance to amodiaquine plus sulfadoxine-pyrimethamine: mutations in pvdhfr and pvmdr1. J Infect Dis. 2008;198(3):409-17.

44. Khattak AA, Venkatesan M, Khatoon L, Ouattara A, Kenefic LJ, Nadeem MF, et al. Prevalence and patterns of antifolate and chloroquine drug resistance markers in Plasmodium vivax across Pakistan. Malar J. 2013;12:310.

45. Ariey F, Witkowski B, Amaratunga C, Beghain J, Langlois AC, Khim N, et al. A molecular marker of artemisinin-resistant Plasmodium falciparum malaria. Nature. 2014;505(7481):50-5. 
46. Ashley EA, Dhorda M, Fairhurst RM, Amaratunga C, Lim P, Suon S, et al. Spread of artemisinin resistance in Plasmodium falciparum malaria. N Engl J Med. 2014;371(5):411-23.

47. Pearson RD, Amato R, Auburn S, Miotto O, Almagro-Garcia J, Amaratunga C, et al. Genomic analysis of local variation and recent evolution in Plasmodium vivax. Nat Genet. 2016;48(8):959-64.

\section{Tables}

Tables 4 and 5 are not available with this version.

\section{Figures}

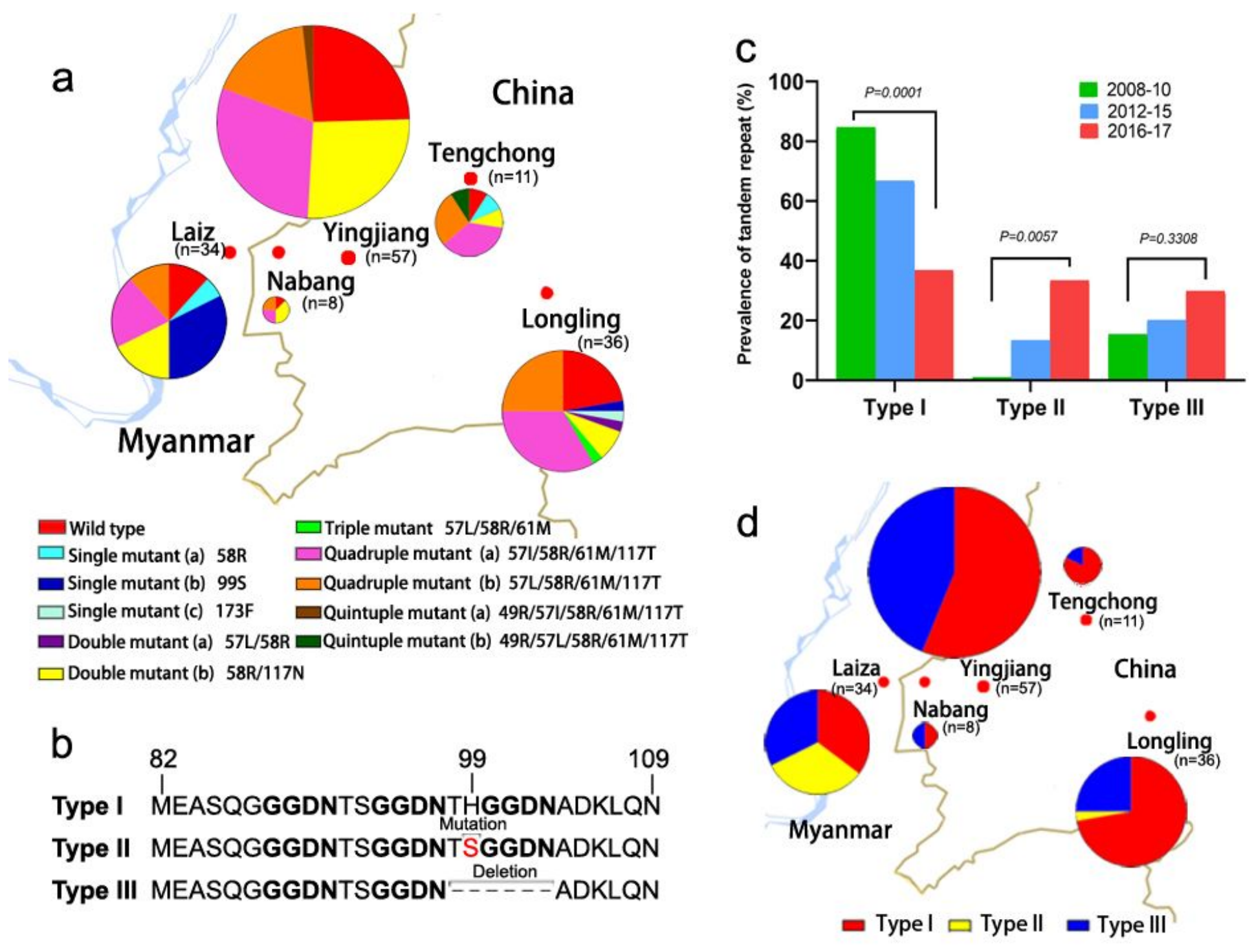

Figure 1

Geographical prevalence of $p v d h f r$ haplotypes and tandem repeat variants. a, The geographical distribution of $p v d h f r$ haplotypes. $\mathbf{b}$, Amino-acid sequence alignment of three tandem repeat variants. Bold letters indicate the tandem repeat. c, Frequency changes of the tandem repeat variants over time. $\mathbf{d}$, 
The geographical distribution of tandem repeat variants. The size of the pie represents the number of the isolates $(n)$.

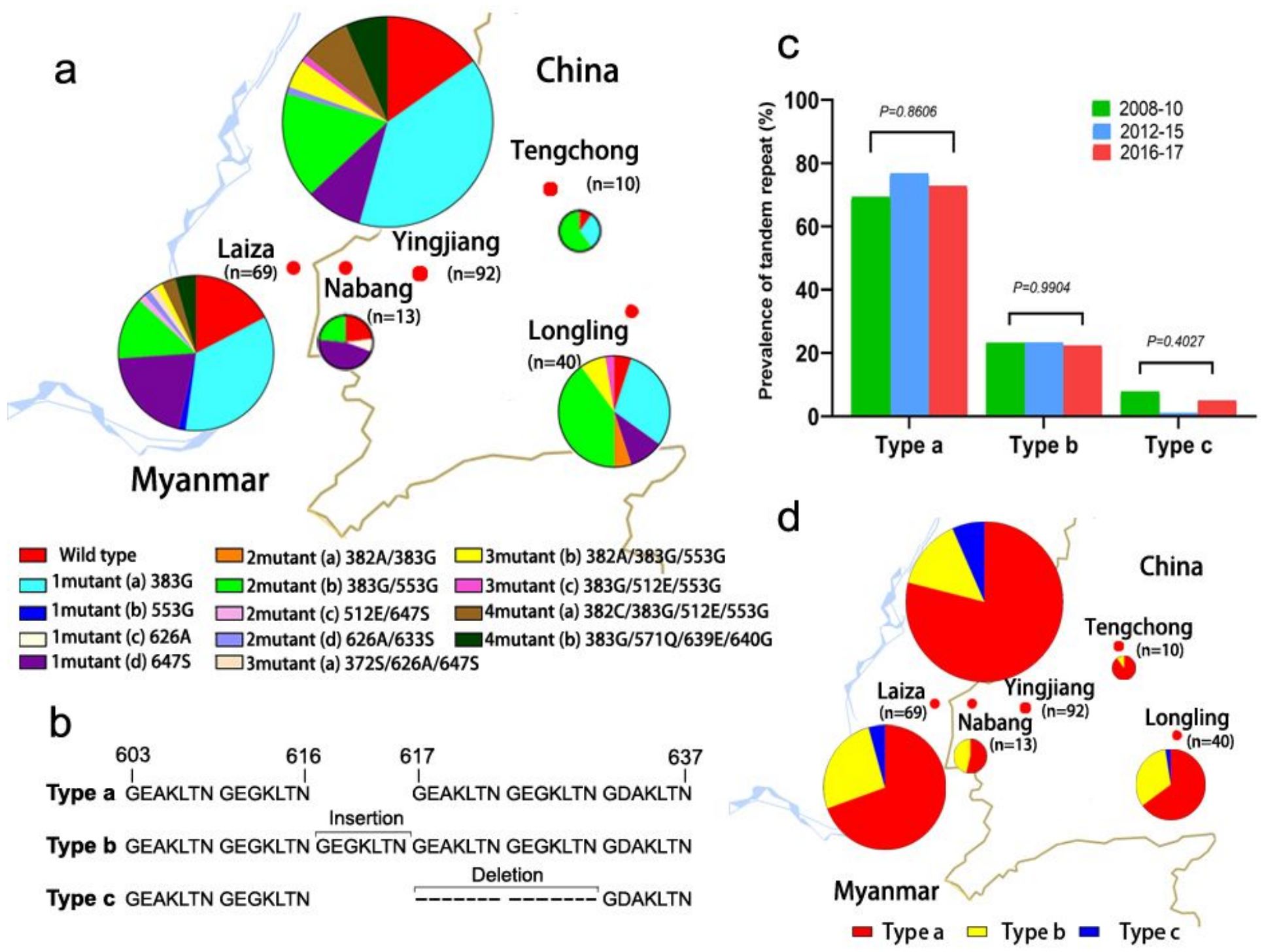

Figure 2

Geographical prevalence of $p v d h p s$ haplotypes and tandem repeat variants. a, The geographical distribution of $p v d h p s$ haplotypes. $\mathbf{b}$, Amino-acid sequence alignment of three tandem repeat variants. $\mathbf{c}$, Frequency changes of tandem repeat variants over time. $\mathbf{d}$, The geographical distribution of tandem repeat variants. The size of the pie represents the number of the isolates (n). 

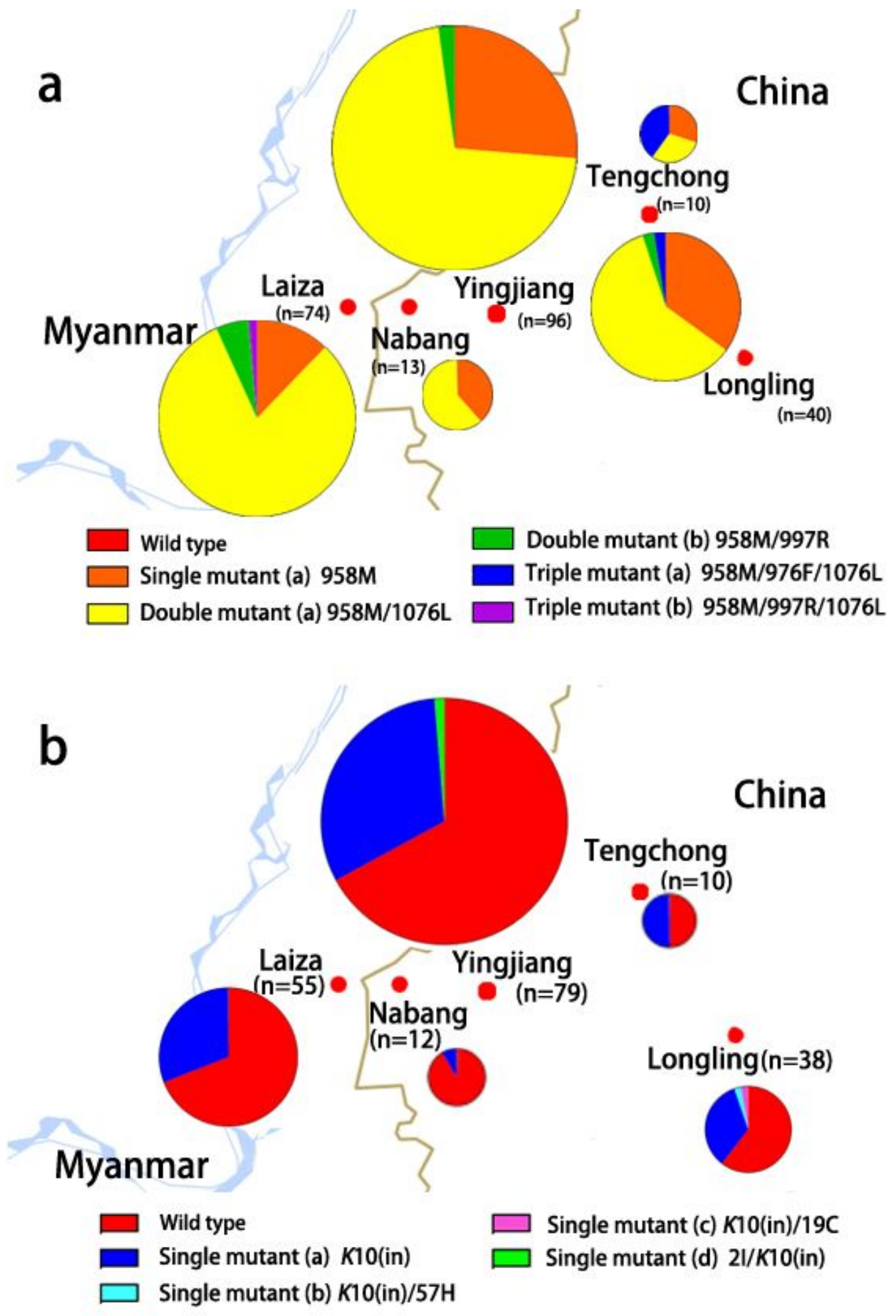

Figure 3

Geographical prevalence of pvmdr1 haplotypes (a) and pvcrt-o haplotypes (b). The size of the pie represents the number of the isolates (n).

\section{Supplementary Files}


This is a list of supplementary files associated with this preprint. Click to download.

- Graphicalabstract.jpg

- Additionalfile1.docx 